\title{
COMMISSION 19: ROTATION OF THE EARTH
}

\author{
(ROTATION DE LA TERRE)
}

\author{
PRESIDENT: Dennis D. McCarthy \\ VICE-PRESIDENT: Nicole Capitaine \\ ORGANIZING COMMITTEE: F. Arias, G. Beutler, P. Brosche, A. Brzezinski, \\ V. Dehant, M. Feissel, R. Gross, S. Manabe, L.V. Morrison, L.V. Rykhlova, \\ V. K. Tarady, J. Vondrak, C. Wilson, F. Yang \& Y.S. Yatskiv
}

\section{INTRODUCTION}

The triennial report of Commission 19 was composed from the contributions of its members. Space does not permit a listing of their names, but their contributions are sincerely appreciated. Unfortunately because of limited space it is also not possible to provide in this report the extensive list of publication of the Commission members. The list of publications is however available on the Commission 19 web site at maia.usno.navy.mil/iauc19.

During the interval 1997-1999, Commission 19 has been very active. The membership rolls were updated and a web site was created containing member addresses, reports, meeting notices and publications of interest. Working Groups associated with the work of the Commission were active, and their reports are contained in this report. The Commission also sponsored two colloquia: "Polar Motion: Historical and Scientific Problems," and "Towards Models and Constants for Sub-Microarcsecond Astrometry."

Reports of various institutions are given in this report, but some accomplishments of particular interest deserve special mention. The International Earth Rotation Service began an extensive reorganization to better meet the needs of its users. The use of polar motion data derived from the analysis of Global Positioning System satellites provided through the International GPS Service grew to the point that these data now provide the most important contribution to the IERS determination of polar motion. The improvement of the precision of Earth orientation observations and the improvement in the frequency of the observations made it possible to investigate high-frequency (diurnal and sub/diurnal) variations in the Earth's orientation. This new area of investigation also prompted discussion on the need for an improved definition of the Celestial Ephemeris Pole in order to clarify the definition of polar motion and nutation. Improvements in observational procedures have also made it possible to obtain estimates of Earth orientation parameters with much improved timeliness. It is also noteworthy that significant progress has been made in the investigation of non-rigid Earth nutation theory.

\section{REPORT OF THE INTERNATIONAL EARTH ROTATION SERVICE}

\subsection{IERS Reorganization}

The IERS Directing Board organized an evaluation of the service to review current operations and solicit advice on future needs for global references. The 1996 IERS Workshop was dedicated to this review. Draft reports and recommendations were discussed during the workshop and finalized by topic coordinators. The IERS Directing Board began implementation of the recommendations at and between the two Directing Board meetings held in October and December, 1996. Major new aspects of the IERS are: maintaining the International Celestial Reference Frame (ICRF), monitoring global geophysical fluids, monitoring the motion of the Earth's center of gravity, organizing interaction with the Working 
Groups set up by IAU, IAG and IUGG on topics relevant to IERS missions., strengthening the development and maintenance of the IERS Conventions, and developing or improving technical abilities for the combination of products.

A decade after the beginning of its operation, the IERS began its reorganization following these recommendations and the reorganization of the contributing techniques (GPS, Laser tracking to the Moon and artificial satellites, and VLBI) into international services. Consideration was also given to improved services, more visibility to the various components of the IERS and opening the service to scientific groups which had developed since the beginning of the IERS. New terms of reference were adopted in mid 1999, and the new structure will be set officially in the beginning of 2000 .

\subsection{IERS Technical Notes}

The Technical Notes published over the period 1996-1999 are: No 21: D. D. McCarthy (ed.) IERS Conventions (1996), No 22: C. Reigber and M. Feissel (eds.) IERS missions, present and future. Report on the 1996 IERS Workshop, No 23: C. Ma and M. Feissel (eds.) Definition and realization of the International Celestial Reference System by VLBI Astrometry of Extragalactic Objects, No 24: C. Boucher, Z. Altamimi, P. Sillard (eds.) Results and Analysis of the ITRF96, No 25: J. Ray (ed.) Analysis Campaign to Investigate Motions of the Geocenter, No 26: D. Salstein, B. Kolaczek, D. Gambis (eds.) The impact of El Niño and other low-frequency signals on Earth rotation and global Earth system parameters, No 27: C. Boucher, Z. Altamimi, P. Sillard (eds.) The 1997 International Terrestrial Reference Frame (ITRF97).

\subsection{The IERS Central Bureau}

Nonlinear time series analysis techniques were applied to daily observations of the length of day (LOD) and polar motion components spanning more than 27 years. The study indicates the chaotic nature of the Earth's rotational dynamical regime (Frede and Mazzega 1998a, 1998b). Horizons of 11 days for LOD, and about 8 to 9 days for polar motion are found beyond which prediction errors will be of the order of the rms of the filtered EOP series.

In the normal-point series $\mathrm{C} 02$ and $\mathrm{C} 03$, the solution at the central dates of intervals is made using a least-squares fit for all EOP components. New algorithms were defined (Bougeard and Caquineau, 1999) for solving both L1 and Huber-M fits that were based on convexity methods and proximal decomposition tools. They were successfully applied to the analysis of minor planets from the Hipparcos data (Bougeard et al., 1997) and in the IERS analyses (Bougeard et al., 1999).

The published near real-time Universal Time has been extrapolated from VLBI determinations. Rapid availability of GPS LOD solutions allows significant improvements if no recent VLBI UT1 determination is available. This yields a better UT prediction. The GPS LOD estimates are integrated and corrected for long-term systematic variations (Gambis 1996). The improvement is about an order of magnitude for a 5-day time lag.

\subsection{The IERS Sub-Bureau For Rapid Service and Predictions}

The IERS Sub-bureau for Rapid Service and Predictions at the U. S. Naval Oservatory continues to combine contributed results from a variety of techniques, observing networks, and analysis groups to form a homogeneous daily time series updated daily and distributed twice weekly as "IERS Bulletin A." The functions of the Sub-bureau are performed by personnel of the Earth Orientation Dept. at the U.S. Naval Observatory on behalf of the IERS. In recent years, the "Bulletin A" polar motion results have been dominated by the precise, daily determinations of the International GPS Service (IGS) combined Final products, with their Rapid series being used for the most recent measurements (Mireault et al., 1999). The Rapid determinations are important for "Bulletin A", providing high-quality results which are most significant for the polar motion predictions needed by real-time users. 
The accuracy of these series during 1997 was estimated to be about 0.1 mas (per component) for the Finals and 0.2-0.3 mas for the Rapids. Implementation on 1 March 1998 of the much more robust and improved terrestrial reference frame realization proposed by Kouba et al. (1998), where the coordinates and velocities of 47 sites are constrained to their ITRF96 values, produced significantly more stable polar motion results. Indications are that the accuracy of the IGS Rapid polar motion values was improved to about 0.15 mas. This, in turn, has improved the quality and reliability of near-term polar motion predictions.

"IERS Bulletin A" has become increasingly reliant on GPS-based estimates for LOD. The IGS started producing an official LOD product on 2 March 1997 using a weighted combination of LOD results submitted by each IGS Analysis Center (AC). To calibrate LOD biases, each series is compared with the most recent 21 days of non-predicted UT1 values from "Bulletin A" (Kouba and Mireault 1997; Ray 1996). Shortly after its advent, the IGS combined LOD results were introduced into the "Bulletin $A$ " combination to extend the UT1 value of the most recent VLBI determination forward by integration. A few months later an independent set of GPS-based estimates of universal time, derived at USNO (Kammeyer, 1999), were also included in "Bulletin A". About three weeks of the most recent estimates are used, after calibration in offset and rate compared to overlapping UT1 results from VLBI. In April 1998, an analogous universal time series from the EMR AC (Natural Resources Canada) was added in a similar manner. The EMR analysis strategy differs from the other IGS groups in applying a priori orbit constraints that allow both universal time and LOD to be estimated simultaneously. These three series together have proven very successful in extending UT1 results forward from the latest VLBI determinations, which can have a latency of up to about a week. As a consequence, the last non-predicted UT1 value in "Bulletin A" is now generally more accurate than 100 microseconds, usually considerably more so.

Martin Mur et al. (1998) have stressed the need for improved EOP predictions for use in computing the IGS Predicted orbits. Partly to address this concern, refinements were implemented in "Bulletin A" in early 1998 to enforce a smooth transition from the last observations to the long-term predictions (Ray and Luzum 1998). The improvement was most significant for the shortest prediction intervals ( $53 \%$ for 1 day) with diminishing effect over longer spans. About a year later, the Rapid polar motion rate determinations provided by the IGS began to be used for the latest epoch only to further improve near-term predictions.

For real-time users, given two updates of "Bulletin A" each week, the longest prediction interval is 7 days. This means that real-time users can experience polar motion prediction errors up to $\sim 2.4$ mas (in an rms sense per component) and UT1 errors as large as $\sim 1$ ms (15 mas). Predictions of UT1 variation are more problematic because the geophysical excitation is about an order of magnitude larger than for polar motion. To reduce these prediction errors significantly requires more frequent "Bulletin A" updates, preferably done daily shortly after the IGS Rapid products are released. Such a process was implemented during 1998 and reduces the longest prediction interval from 7 days to $\sim 58$ hours, under normal circumstances. Now the maximum errors for real-time users should be less than $\sim 0.7$ mas (RMS per component) for polar motion and less than $\sim 0.2 \mathrm{~ms}$ for UT1.

All results are available at <http://maia.usno.navy.mil/>. Included are statistical summaries as well as plots of residuals for each of the contributing EOP series. Additional analysis reports are prepared occasionally to assess performance changes or to evaluate the geophysical implications (e.g., Eubanks et al., 1998).

\subsection{Special Bureau For the Atmosphere}

The Special Bureau for the Atmosphere, formerly entitled the Sub-bureau for Atmospheric Angular Momentum (AAM), intercompared analysis data sets and assessed the skill of forecasts of the relevant atmospheric quantities. The basic SBA parameters are derived from the operational analyses of major weather centers. In addition, meteorological centers reanalyze the state of the atmosphere over long periods, applying consistent modern 
meteorological data assimilation systems to historical observations. By the end of 1998 , 51 years (1948-1998) of such reanalyses had been performed by the United States National Centers for Environmental Prediction, and nearly 16 years (3/1980-11/1995) by the NASA Goddard Space Flight Center Laboratory for Atmospheres. Also, the European Centre for Medium Range Weather Forecasts has finished its 15 year (1979-1993) reanalysis, and plans to complete the 40-year assimilation in about two years. AAM quantities from these reanalyses are being computed by SBA personnel and associates. Another mission of the SBA is to interpret the larger scale interactions of the atmosphere on geodetic parameters. They have examined the influence of the El Niño/Southern Oscillation (ENSO) on AAM though a modification of the zonal wind structure of the globe.

The calculation of wind contributions involves the computation of integrals over the depth of the atmosphere, which varies in the global models. Therefore, wind contributions, effectively to $100 \mathrm{hPa}$, as well as to the top of each model, were specified so that values from the various centers could be compared more accurately. The pressure-related components from the centers can be compared without regard to the vertical construction of the model. A key issue is the extent to which an equilibrium response of the ocean modifies the changes in the pressure distribution felt by the solid Earth. At certain time scales, this inverted barometer (IB) response acts to reduce the equivalent angular momentum fluctuations of the atmosphere. Because the IB exists to at least a certain extent, calculations with this IB correction are performed in parallel to the regular pressure terms. Other meteorological parameters are included in the SBA files. Zonal mean zonal wind, from which the $\chi_{3}$ term can be calculated, and zonal mean temperatures, whose gradients are related to overall zonal winds, are SBA analysis fields. Routine specification of the mass distribution of the atmosphere, expressed in low-order spherical harmonic expansions, is important in the study of various problems in geodynamics and geodesy, including the effects of mass redistribution on satellite orbits. These coefficients are also SBA fields.

Effective angular momentum functions from the four operational analyses and from the NCEP/NCAR reanalysis system are available from the SBA Web page from AER, at http://www.aer.com/groups/diag/sb.html. These variables are available in near-real time, from the major weather centers and are maintained, in both analysis and forecast form, for at least the past 10 days, by anonymous ftp from the NCEP server at nic.fb4.noaa.gov under subdirectory pub/nws/nmc/cac/htdocs/products/stratosphere/sbaam. The data are updated on a daily basis. Additional parameters are available from AER, by request, to salstein@aer.com.

The torques that effect the transfer of angular momentum between Earth and atmosphere are being made available through the SBA website. These relate to the pressure gradient forces against mountainous topography (mountain torque), and tangential forces against the land and ocean surface (friction torque). Mountain torque has been shown to be related to the rapid fluctuations in total AAM, and in global LOD. Atmospheric systems passing over the Rocky Mountains and Asian orography were particularly important in some episodes of rapid fluctuations in LOD (Iskenderian and Salstein, 1998). Additional torques, based on the NCEP/NCAR Reanalysis, were produced by K. Weickmann of NOAA. Additional files are being prepared at the SBA for research purposes. Forecasts of zonal mean zonal winds out to the longest forecast times is one file. Analyses using several definitions of the world ocean to calculate the inverted barometer correction forms two other files. These quantities were prepared for a limited period.

Currently, there are four operational participating centers in the Sub-bureau and each produces a portion of the requested data. The centers include the U.S. National Centers for Environmental Prediction (NCEP), formerly National Meteorological Center (NMC), the United Kingdom Meteorological Office (UKMO), the European Centre for MediumRange Weather Forecasts (ECMWF), and Japan Meteorological Agency (JMA). Data are transmitted rapidly to the SBA. The full archive of these centers resides at AER. The NCEP and JMA centers produce alternate series with and without the Inverted Barometer (IB) formulation, in parallel. 
The effort to analyze the state of the atmosphere in a consistent way was performed back to 1948 with an advanced data assimilation system and as much historical meteorological data as were practical to utilize (Kalnay et al., 1996). This system is run at a truncation of 62 triangular waves and 28 levels in the vertical up to near the $10 \mathrm{hPa}$ level. They have undertaken the mission of calculating the global/hemispheric SBA parameters from this reanalysis system based on the four-times daily temporal resolution. Details concerning angular momentum calculations and results from reanalysis are given in Salstein and Rosen (1997). The SBA data products based on both NASA Reanalyses and ECMFW Reanalyses are under preparation and will be submitted to the SBA archives upon completion.

The issue of a model bias is important to deal with, and knowledge of such can help to improve the overall utility of the forecasts. A measure of skill (Salstein et al., 1993) of $\chi_{3}$ wind is positive for all lead times. Coherence analysis shows significant agreement on time scales down to about 6 days. Further work is being done now to include the influence of oceanic forcing on polar motion (e.g. Ponte et al., 1998) which is considerable, particularly on seasonal timescales. Geographical areas whose atmospheric pressure fluctuations appear especially important for polar motion are the continental regions of Eurasia, and to a lesser extent, North America (Nastula and Salstein, 1999).

\subsection{Sub-Bureau of the Coordinating Center for Monitoring Global Geophys- ical Fluids (MGGF) of the IERS.}

V. Dehant is the coordinator of the Special Bureau for the Core, inside the Subbureau of the Coordinating Center for Monitoring Global Geophysical Fluids of the IERS. A web site has been created (www.oma.be/KSB-ORB/SBC/) including information about the link between the core and Earth's rotation, and about core angular momentum. It also provides a bibliography associated with these topics.

\section{REPORT OF THE WORKING GROUP (WG) ON NON RIGID EARTH NUTATION THEORY}

The WG on Non-rigid Earth Nutation Theory worked by e-mail. They separated the discussions into 6 different levels. The first level concerned the seismic input models used to compute the transfer function for nutations. The points examined were the validity of the PREM model, the violation of the hydrostatic equilibrium to reconcile the computed and observed moments of inertia, the dynamical flattening and the boundary. The second level concerned the transfer function for nutations. These incorporate changes in the boundary shapes due to non-hydrostatic equilibrium, changes in density which provide the observed hydrodynamical flattening and changes in elastic parameters to incorporate the effects of mantle inelasticity. The third level concerns rigid Earth nutation theories. Scientists of this discipline converged to series very close to one another. The fourth level concerned the convolution between the transfer function and the rigid Earth nutation series. The WG discussed the coherence between the constants used in the transfer function and the rigid Earth nutation series and the accuracy of the results of this convolution. The fifth level concerned the ocean and atmospheric effects on nutation. The angular momentum and torque approaches were used. They also considered the indirect effects of the atmosphere and pointed out the necessity of having an atmospheric data model that provides a correct estimation in the diurnal frequency band and to have dynamic ocean models in the same frequency band. The sixth level concerned comparison with the observations. They stressed the necessity of having continuous VLBI observations. The questions and answers were published in Celestial Mechanics (in press). The WG also organized joint discussions at the IAU General Assembly and special sessions at the Journées Systeme de Reference. 


\section{REPORT OF SUBGROUP T5 "COMPUTATIONAL CONSEQUENCES" OF THE IAU WG ON ICRS}

The consequences for precession, nutation and Earth rotation of the adoption of a new celestial reference system (ICRS) by the IAU on 1 January 1998 is under study within the subgroup T5 "Computational Consequences" (chair: N. Capitaine) of the IAU WG on ICRS from 1998. Its work includes the consideration of the most relevant Earth orientation parameters (EOP) to be used to represent Earth rotation with the best accuracy in the ICRS (Capitaine \& Guinot 1997, 1999, Capitaine 1998a, Capitaine et al. 1999) and the need for a new definition of the Celestial Ephemeris Pole (CEP) consistent with current theory and observations (Capitaine 1998a, 1998b; Capitaine \& Brzezinski 1999).

\section{REPORTS FROM INDIVIDUAL INSTITUTIONS}

\subsection{Space Research Center, Warsaw, Poland}

Stochastic models of polar motion in algorithms to process and interpret Earth orientation data (Kalman filter, least-squares collocation) were investigated. Theoretical studies and numerical experiments proved the superiority of stochastic over classical methods. Phenomena studied in detail were atmospheric normal modes (Petrov and Brzezinski 1996; Petrov et al. 1997; Petrov 1998). A maximum likelihood method (MLM) algorithm for direct determination of the Free Core Nutation (FCN) parameters was used to analyze the FCN signal in the celestial motion of the pole observed by VLBI. The observations yield the FCN period estimate $T=425.9 \pm 7.9$ days. The MLM estimate of the FCN quality factor is $\mathrm{Q}=40280$ with $1-\sigma$ confidence interval $(13710, \infty)$. Comparison with the AAM data shows that nearly diurnal variations of the surface pressure have enough power to excite the FCN, but the wind contribution is negligible (Brzezinski 1996; Brzezinski and Petrov 1998). Estimation of the atmospheric contribution to nutation amplitudes was extended to diurnal and semidiurnal bands in polar motion and LOD (Petrov 1998; Petrov et al. 1998a). Comparison with VLBI nutation data shows that the global ocean circulation model used in the analysis is not realistic in the diurnal band, but such a comparison provides important information on how to improve this model (Petrov et al. 1998b).

Theoretical investigations concerning geophysical interpretation of modern Earth rotation observations and the related geophysical data were continued including the definition of the Celestial Ephemeris Pole and the equation of polar motion (Brzezinski 1998). Chandler and semi-Chandler oscillations were studied, and the semiannual oscillation and the semiChandler wobble were separated using the Fourier Transform Band Pass Filter (FTBPF). Long-period variations of their amplitudes with the period of 11-14 years were demonstrated. The correlation coefficient between the change of amplitude of the semi-Chandler wobble and the Wolf numbers is significant at the $90 \%$ confidence level. It was found that the temporal variations with a period of 3-4 years of the amplitude of the semiannual oscillation filtered using a wide band pass filter are simply beats from the two oscillations (Kosek and Kolaczek 1995a; 1995b; 1996, 1997). Oscillations with periods of $75,40,30,20$ years were detected in variations of the Chandler nutation amplitude. The oscillation with the period of 40 years is the most energetic and suggests the influence of variations of the magnetic field which have similar oscillations. Regional values of the effective AAM were obtained from the Japanese Meteorological Society (Nastula 1995, 1997, 1997; Nastula et al. 1998b; Nastula and Kotreleva 1998a) and from a nearly 40-year-long data series from the NCEP/NCAR Reanalysis Project (Nastula and Salstein 1998a, 1998b, 1998c). In cooperation with D. A. Salstein from the Atmospheric and Environmental Research Inc (AER), ways in which different regions are responsible for excitations of high frequency polar motions were explored using a much longer and more reliable data set than was previously available (Nastula and Salstein 1998a, 1998b, 1998c). The Eurasian region was isolated, as particularly important for exciting high-frequency polar motion (Nastula and Salstein $1998 \mathrm{a}, 1998 \mathrm{~b}, 1998 \mathrm{c}$ ). It is clear, however, that the atmospheric data do not explain all of the observed high-frequency polar motion signals. The addition of oceanic excitation to at- 
mospheric excitation leads to substantial improvements in the agreement between observed polar motion at seasonal and intraseasonal periods (Nastula et al. 1998c; Nastula and Ponte 1999). To estimate equatorial excitation functions for the ocean they used velocity and mass fields from the constant density ocean model by R. M. Ponte from AER. Analysis of the influence of shallow seas and shelf areas on the variations of AAM was performed. It was concluded that from 10 to 150 days, the maximum contribution from seas to the standard deviation of AAM could be about $40 \%$ (Nastula and Manabe 1997; 1997b). The changes of the amplitude of the AAM function are 100 to 300 times smaller than the changes of the shallow and shelf sea regions (Nastula and Manabe 1997, 1997b). The correlation of El Niño phenomena with the influence of AAM on polar motion was analyzed. Correlation coefficients between two El Niño parameters, differences of sea surface temperatures and sea surface atmosphere pressures in different regions of the Pacific Ocean and variations of correlation coefficients between AAM and polar motion were computed for several El Niño phenomena. El Niño disturbs correlation coefficients between the AAM and polar motion and causes impulsive irregular variations of short-period oscillations of polar motion (Kosek et al. 1995c; Kolaczek et al. 1998a, 1998b; Nuzhdina et al. 1997). Short-period oscillations with periods of $182,120,90,60$ and 30 days in sea level anomalies determined from altimetric measurements of TOPEX/Poseidon were detected. These oscillations have amplitudes ranging from $10 \mathrm{~cm}$ in the case of the semiannual oscillation to $2-3 \mathrm{~cm}$ for the shortest ones (Kolaczek et al. 1997). Amplitudes of these oscillations vary with geographic latitudes.

\subsection{Observatorium Hoher List, Univ. Sternwarte Bonn}

In studies of oceanic angular momentum (OAM) they went from climatological variations to the instantaneous variations for the last decades of precise VLBI observations, starting a model which treats simultaneously the tides and the general circulation of the oceans, including the interaction with the atmosphere. Tides are accounted for with their total instantaneous values and not decomposed into various frequencies. A more popularized version of an earlier article now has the title P. Brosche "Understanding tidal friction; A history of science in a nutshell" appeared in the electronic journal "Science Tribune" Paris, in December 1998 (http://www.tribunes.com/tribune/art 98/bros.htm)

\subsection{The Institute of Physical Geodesy, Univ. of Technology, Darmstadt}

They investigated details affecting Earth rotation in connection with core-mantle boundary effects, mantle anelasticity, long-period nutation, inner core stratification and fluid outer core structure.

\subsection{Paris Observatory Department of Fundamental Astronomy}

New tables REN-2000 (Souchay et al. 1999) corresponding to the analytical development of nutation for a rigid Earth model were constructed using a Hamiltonian approach. The nutations of the axis of angular momentum, axis of figure and axis of rotation were provided. The model includes all effects truncated at 0.1 microarsecond including the second-order effects due to cross nutation and spin-orbit coupling, as well as diurnal and sub-diurnal components from the harmonics of degree 2, 3 and 4 of the geopotential (Folgeira et al. 1998). The accuracy of the tables is of the order of 1 microarsecond in the frequency domain and 10 microarseconds in the time domain (Souchay 1998). The influence of the atmosphere on precession-nutation was studied using the angular momentum approach as well as the torque approach. The use of a 29-yr time series of atmospheric effective angular momentum provided the amplitudes of the most important atmospheric contributions to the annual prograde and semiannual nutations as well as for the constant offset of the pole (Bizouard et al. 1998). The contribution of the atmosphere to nutation has been estimated from the numerical computation of the atmospheric torque acting on the solid Earth using 40 years of atmospheric "reanalysis project" provided by the US NCEP and NCAR (de Viron et al. 1998). A combination of 8 years of superconducting gravimeter data from 
Strasbourg and VLBI Earth orientation data was used to determine the gravimetric factor at the Chandler period (Loyer et al. 1999). The results are in good agreement with the longperiod theoretical gravimetric factor for an elastic solid Earth model and static pole tide in the oceans. The atmosphere has been shown to perturb the amplitude of the Chandler wave observed in the gravimetric data.

\subsection{Activities Related to Earth Rotation in China}

There are 5 fixed SLR stations located in Shanghai, Changchun, Beijing, Wuhan and Kunming in the Chinese SLR Network. The Kunming station operated routinely since December, 1998 (Zhang et al. 1998). The equipment of all five stations was upgraded in the last two years (Yang, et al. 1998). The Changchun station is able to get more then 2600 passes per year (Zhao, et al. 1998). The Shanghai station has daylight tracking capability (Yang et al. 1999) and a multi-satellite alternate tracking and control system was developed. The track rail of the 25-meter telescope and the encoders at Shanghai VLBI Station were repaired in 1997-1998. For the Urumqi VLBI station, a dual feed for S/X bands was redesigned and rebuilt in 1998. The new cooled X-band receivers from IAA (Russia) were operational at both stations since 1997 . A $40 \mathrm{~K} \mathrm{~S}$-band receiver was developed at SheShan station in 1999.

Using the $\Delta \mathrm{T}$ series (1891.5-1955.5) derived from the lunar occultation observations by Jordi et al. (1994) and the UT1-UTC series (1955.5-1997.5) of the BIH/IERS, a new $\Delta$ LOD series in monthly intervals from 1892.0 to 1997.0 was calculated. The interannual and decadal components were separated and compared with those inferred from other geophysical quantities. Wavelet analyses of interannual LOD variation, ENSO and atmospheric quasi-biennial oscillation (QBO) from 1964 to 1993 were done. Their magnitude and phase relations clearly show that ENSO and QBO are responsible for most of the interannual LOD variation (Zhou \& Zheng 1997a). The North Atlantic Oscillation (NAO) is probably the most prominent and well-studied interannual meteorological oscillation system besides ENSO. An index (NAOI) used to measure the strength of NAO was compared with the observed interannual polar motion excitation functions during 1964-1994. Studies show significant correlations between them (Chao \& Zhou 1998, Zhou et al. 1998). Based on the polar motion and NAO data since the beginning of this century, the influence of NAO on decadal polar motion was discussed (Zheng et al. 1997). Analyses show that polar motion excitation functions correlate well with NAOI at frequencies where they correlate poorly with ENSO, and vice versa, suggesting that combining ENSO and NAO, as well as other systems, should better explain the observed interanmual polar motion and perhaps the Chandler wobble (Chao \& Zhou 1998). Using LOD series derived from BIH/IERS UT1UTC, the LOD (wind) inferred from the wind term of AAM (NCEP/NCAR) and LOD(SOI) from the index of southern oscillation a comparison was made by Liao on inter-annual time scales during 1968-1997.

The LOD time series and the AAM derived from global meteorological data during 1979-95 by the NMC and the NCEP/NCAR were employed to reanalyze the excitations of LOD, and to examine the contribution of each AAM series to LOD (Yu et al. 1998). Atmospheric excitation function series were compared with the geodetic excitation function derived from the deconvolution of the Space 93 data during 1984-1992. The observed polar motion was also compared with the excitation polar motion derived from the convolution of the atmospheric excitation series (Gu \& Wang 1997). The trend, annual polar motions and Chandler wobble (CW) for every year were determined (Gao 1999).

OAM is a likely candidate among the other excitation sources. The theoretical model and the calculation method of OAM were studied using the 20-layer oceanic model of the Institute of Atmosphere and Physics, Chinese Academy of Sciences. There are obvious annual variations in the OAM series. The integrated series of the axial component of OAM are out of phase in the northern and southern hemispheres. The oceanic excitation of residual annual variation of $\mathrm{LOD}$ is $82 \%$, of the $\mathrm{x}$-component of polar motion $64 \%$ and of the $y$-component $93 \%$ (Yu \& Zheng 1998). Using the TOPEX/POSEIDON data, the global 
and regional sea level changes and their relation with Earth rotation and ENSO phenomena were analyzed (Wu \& Zheng 1997, Zheng \& Wu 1997).

In early 1997 an El Niño event occurred. The change of sea surface height during the event, monitored with TOPEX altimetric data, contributed to the study of the formation and development mechanism of an $\mathrm{El} \mathrm{Niño} \mathrm{event} \mathrm{and} \mathrm{its} \mathrm{impact} \mathrm{on} \mathrm{the} \mathrm{global} \mathrm{climatic}$ change (Dong et al., 1999). A wavelet analysis was applied to study the intra-seasonal frequency spectrum of LOD and correlations with ENSO (Zhong et al. 1998). The Hilbert transform was used to extract the amplitude modulation signals for the intraseasonal LOD from 1962 to 1996 . The results reveal that the intraseasonal LOD amplitude modulation variation has a positive secular trend, and an interannual variation which is ahead of the ENSO evolution (Zhong et al, 1999). The relation between the variation of the Earth rotation rate and earthquakes was studied (Gu 1996, 1997b). A relation between El Niño and seismic activity was found. Seismic activity especially for earthquakes with magnitudes greater then 5.0 could induce sudden jump of the axial moment of inertia of the Earth. The jump corresponds with the peak of El Niño events ( $\mathrm{Gu} 1999$ ).

Using the fault plane parameters, the location of earthquake epicenters and the expression given by Dahlen, the cumulative change of the equatorial moment of inertia of the Earth for the 1977-1994 data in the Harvard Catalogue was estimated (Gu 1997a). Perturbations of the inertia tensor caused by ice melting in the past 21000 years were calculated using parameters of the four biggest ice sheets based on the post-glacial rebound model ICE-4G, and the theoretical direction of secular drift rate of the Earth's pole was found to be along $74.2^{\circ} \mathrm{W}$ (Yang 1999). The mean lower mantle viscosity was estimated with the constraint of the non-tidal acceleration term of the Earth rotation obtained with the results of modern space geodetic techniques (Yang et al. 1998).

A model of resonance excitation as a nonlinear dynamical mechanism of polar motion was proposed. The existence of nonlinear terms may introduce combined frequencies in polar motion (Wang 1998). The CW equation was modified to be the Duffing equation by using a plane nonlinear pendulum model with linear damping and periodic forcing (Wang 1999). The new homogeneous set of polar motion data for the years 1899.7-1992.0, was analyzed (Wang \& $\mathrm{Gu}, 1999$ ).

The components of geocenter variation were obtained from Lageos data (Sept. 1983Dec. 1994) (Wu, et al. 1999). Using the global meteorological monthly data, the solid geocentre motion due to the seasonal distribution of air pressure and ground water was estimated. The solid geocentre motion due to ground water may have a larger contribution than that of air pressure, and the relative maximum value of the motion of the solid geocentre was about $4 \mathrm{~mm}$ (Zhou \& Gao 1998). The $\mathrm{K}$ Love numbers were determined from UT1 series from 1962.0 to 1982.0 by Li Zhengxin. The solid Earth tide Love number and phase lag using space geodetic data were studied (Wu et al., 1997, 1999). The low degree geopotential variation, ocean tides, secular and decade change of LOD were discussed (Peng 1998; Wu, et al., 1998). With the rheological parameters given by the PREM model, the tidal potential of the Earth's rotation due to the zonal tides in the form of a set of coefficients was obtained (Xiao Naiyuan, et al., 1997).

The secular variation of the Earth's rotation derived from observations of ancient astronomical phenomena was studied. Possible variation of non-tidal factors in the Earth's rotation was discussed (Han 1997). The interaction between the solar wind and the magnetosphere has an effect on the long-term variations of Earth rotation producing an angular deceleration. This result may lead to a search for other sources of the secular variation of the rate of the Earth's rotation variation to reconcile the observed and the theoretical results ( $\mathrm{Gu}$ 1998). The Hamiltonian method was applied to calculate the Earth's deformation. Taking the viscous elasticity into account, the secular deceleration and its effect on variations of the Earth's rotation were calculated (Xiao et al. 1998).

Temporal variations of forced nutation terms can be distinguished (Li \& Zheng 1997). Corrections to the IAU 1976 precession constant and the coefficients of the IAU 1980 nutation series were determined from VLBI observations. (Li \& Wang 1998). The rheological 
parameters for the Earth model PREM were adopted to study the rotational motion of an elastic Earth. The Hamiltonian theory was applied to calculate the nutation series for the figure axis (Xia, et al., 1997). Recent progress in the study of precession and nutation was discussed and the FCN period was determined (Zhang 1999).

The possibility of detecting the signal of Earth rotation with super conducting gravimeters was discussed (Huang et al., 1998). The inverted barometer using air pressure and altimeter data was studied and its effect on Earth rotation was discussed (Yan Haoming 1999). The strict formula of gravity variation caused by the movement of the instantaneous rotation axis was determined. The stability of the solution for the coordinates of three observing stations and four retro-reflectors using Lunar Laser Ranging was also investigated.

\subsection{Royal Observatory of Belgium}

The new model of rigid Earth nutations (Roosbeek and Dehant 1998) includes the classical $\mathrm{J} 2$ terms due to the Sun and the Moon, terms due to J3 and J4 for the Moon, direct and indirect planetary effects, lunar inequality, J2 tilt, planetary tilt, effect of precession and nutations on the nutations, secular variations of the amplitudes, effects of the Earth's triaxiality, additional out-of-phase terms due to second order effects and relativistic effects. New transfer functions for nutations and for LOD variations induced by the zonal tides were obtained from numerical integration inside the Earth, modeled as a three-layer body. This non-hydrostatic model of the Earth has core flattening in agreement with the observed FCN period and a dynamical flattening in agreement with the observed precession constant. The next step in this study will be to include viscous and electromagnetic couplings at the core-mantle boundary (Dehant and Defraigne 1997, Defraigne and Smits 1999).

It has been shown that there is some energy near the FCN and the FICN periods in the spectrum of the residuals between the observed nutations and the empirical nutation model of Herring (given by the IERS Conventions), if the FCN free mode proposed by that model is ignored. This energy is not constant in amplitude as well as in frequency, and it can be explained by atmospheric forcing. It was shown that the period of the FCN is stable. The variations between 431 and 434 days are within the limits of uncertainty of the VLBI data.

\subsection{Main Astronomical Observatory, Kiev, Ukraine}

A. Korsun, G. Kurbasova (Crimea Astrophysical Observatory), and J. Vondrak compared different series of Earth orientation. Three different series were considered: the classical optical astrometry series in the HIPPARCOS system, the ILS series of polar motion 19001979, and the IERS combined solution. A. Korsun and G. Kurbasova analyzed the secular variation from different series of polar coordinates. A. Korsun and Ya. Yatskiv analyzed the re-reduced latitude and time observations based on the HIPPARCOS frame in connection with the problem of the existence of secular polar motion. It is shown that there are nonpolar variations of mean latitude and longitudes which could hamper the determination of secular polar motion. The Main Astronomical Observatory National Academy of Sciences of Ukraine organized the Fedorov Memorial Conference in Kiev, June 27-29, 1999. CoChairmen of the Organizing Committee were Ya. Yatskiv and A. Korsun. The proceedings of the conference will be published in 1999.

The series of CRF RSC(GAO UA) have been compiled applying the Kyiv arc method to initial frames derived from VLBI data. The latest version RSC(GAO UA)98 C 01 was published in Kinematics and Physics of Celestial Bodies, 14, 1998. The problem of the search for an exitation mechanism of the Chandler Wobble was briefly reviewed and the resonance exitation of electromagnetic nature was discussed. 


\subsection{Romanian Astronomical Activity Concerning the Earth's Rotation}

Papers were published dealing with satellite dynamics in the gravitational field of the rotating Earth. The post-Newtonian perturbations caused by terrestrial rotation (the LenseThirring effect) were especially studied. The stability of the motion under such conditions was also approached. Concern for the future of classical ground-based astrometry was discussed within the framework of a workshop chaired by Dr. Magda Stavinschi. This workshop was held at the Journées "Systemes de reference spatio-temporels" 1997 (organized by the Astronomical Institute of the Romanian Academy in cooperation with Observatoire de Paris and the Astronomical Institute of the Academy of Sciences of the Czech Republic), 22-24 September 1997, Prague. Among other problems, the usefulness of the astronomical observations using classical instruments for the determination of the Earth's rotation was analysed. Such contributions are considered for maintaining a regional reference frame.

\subsection{Royal Greenwich Observatory and University of Durham}

L. Morrison (RGO, UK) and F.R. Stephenson (Durham, UK) have continued their research into long-term variations in the rate of rotation of the Earth using historical accounts of eclipses, both solar and lunar, back to about $700 \mathrm{BC}$. All the data were published and discussed by Stephenson in Historical Eclipses and Earth's Rotation, Cambridge University Press, 1997. Morrison \& Stephenson discussed the non-tidal components in the Earth's rotation. With J.E. Jones (Proudman Oceanographic Laboratory, UK) they refined the analysis of the eclipse of AD 1567 observed by Clavius, and derived an accurate value of Delta $T$ at that epoch. With J. M. Steele (Durham) they discussed the accuracy of eclipse times measured by the Babylonians and came to the conclusion that they could read their clocks with a mean accuracy of 8 mins and that the accuracy remained the same throughout the Late Babylonian period.

\subsection{Activities Related to Earth Rotation in Russia}

The contemporary accuracy level of determining Earth rotation parameters requires a theory more sophisticated than the widely accepted dynamic theory that considers Earth rotation relative to its mass center independently of the movement of the mass center around the Sun. Bondarenko et al. (1998) proposed a new mathematical model for a solid elastic Earth that dissipates energy by deformations, and performs orbital-rotational motion in the Earth-Moon system in the gravitational field of the Sun. Rykhlova et al. proposed that these forces might generate non-linear oscillations that influence the frequencies of the oscillations. A numerical simulation of polar coordinate data from 1962 to 1992 revealed two basic oscillations with periods $\mathrm{P} 1=408 \pm 5$ days and $\mathrm{P} 2=82 \pm 5$ days. The first value approximates the value of the free oscillations of the Earth, the frequency of the second represents one third of that for the Moon's orbital motion.

The search for correlation between polar coordinates and other geophysical and heliophysical processes continued. The same authors analyzed data concerning Sun spots, polar coordinates variations of LOD, seismic energy, and the index of southern oscillations for the period from 1900 to 1989 . Common variations with a period of $10.4 \pm 0.3$ years were found, and a possible means to synchronize these variations with geophysical and heliophysical processes was shown. Two series of polar coordinates (CIO and HIPPARCOS systems) were compared for frequencies less than the Chandler oscillation frequency (Kurbasova et al. 1997). Variations with periods of 6.4 and 42.67 years were detected. In (Kurbasova et al. 1997) a study of polar motion was carried out by an analysis of the spectral power density function and the Poincare method.

The inability of the present theory to describe completely the observed characteristics of Earth rotation and polar motion is partly due to the fact that the description of tidal effects is based on the classic scheme by Laplace and accordingly on Kepler's two-body problem. In reality the motion of the non-homogeneous Earth is influenced by the Sun, the Moon and 
other planets, so that the Laplace approach can be considered only as a zero approximation. The results of investigations concerning tidal effects for a basic model were applied to study tidal effects in a Sun-Earth-Moon-Planets system (Kurbasova et al. 1998, Gerasimov et al. 1998, Gerasimov and Mushailov 1999, Gerasimov et al. 1999). The authors developed a non-contradictory interpretation of polar motion astrometric observational data. The results are based on a new approach and avoid several shortages of the present theory.

Gravitational lensing of dark bodies was shown to increase the instability of the celestial reference frame. The random rotation of the celestial reference frame limits the accuracy of nutation terms to one microarcsecond. The existence of secular and long-periodic variations of the terrestrial reference frame caused by differential rotation of the crust relative to the mantle is shown. The results are in agreement with LOD observations over the past 2500 years. Non-tidal secular acceleration and quasi-periodic fluctuations on a time-scale of about 1500 years can be explained by rotation of the crust. The approach based on the angular momentum budget of the system Earth-atmosphere was applied to compute the atmospheric contribution to nutation. The main wave corresponding to the annual prograde nutation is the S1 wave. Corrections to several nutation terms were computed; the largest is for the retrograde annual term.

A series of papers by N. S. Sidorenkov was devoted to oscillations of the atmosphereocean-Earth system. Interaction of atmospheric circulation with processes in the ocean creates stochastic oscillations that are superposed by external forces. Several super-harmonics of the main component of the induced nutation movements in the atmosphere and the ocean approach sub-harmonics of the pole's free motion. Polar motion El Niño and La Niña in the ocean, Southern oscillation in the atmosphere and nutation waves are separate blocks of a single phenomenon, the nutation of the Earth-Ocean-Atmosphere system. Presently the construction of models is under way to predict the phase and appearance of El Niño oscillations.

Several papers by Barkin were devoted to a general analytic perturbation theory of the motion of rotating deformed celestial bodies with a description of the perturbed rotation of the Earth. A difference in the eccentricity of the unperturbed pole trajectory for a solid Earth and for an Earth with elastic mantle was detected. The interconnection of the lithosphere with other non-spherical and non homogeneous shells of the Earth and its possible consequences were studied. The variation of the coefficient of the second harmonic of the geopotential due to rotational deformation of the Earth was estimated.

At the Institute for Astronomy (IAA) (Moscow) yearly solutions for x, y, LOD and the coordinates of observing stations with laser ranging data of the LAGEOS satellite were obtained. At the IAA, the software package GROSS is being used to process SLR observations and OCCAM is being used to process VLBI observations. ERA developed by G. Krasinsky and his group is being installed to provide global VLBI solutions. Both operational and yearly final EOP series have been contributed regularly to IERS. The SLR series is also used for computation of the operational IERS solution at USNO. During the last three years significant improvement of software and hardware was completed. As a result, random and systematic accuracy of the series have been improved. Operational SLR solutions with delays of about two days are possible if observational data are available. Another problem under investigation is the computation of a daily independent EOP series based on results obtained with VLBI and satellite techniques.

\subsection{Geoforschungszentrum Potsdam, Germany (GFZ)}

Global GPS data have been analysed by the IGS Analysis Center at the GFZ Potsdam. The excellent data distribution permitted daily resolution in the ERPs. Continuous daily series for an interval of 6 years is available. There are two catagories of products, rapid and final ones. The rapid product is based on an optimized set of about 30 well distributed sites and is computed 12 hours after the end of the day. The final product is derived on a weekly basis, four days after the end of the week, and it uses about 60 sites. 
The accuracy is strongly connected with the accuracy reached in the realization and maintenance of the terrestrial reference frame. This depends on the quality of the GPS stations (data, environment, monumentation) and of the orbit models. In both areas improvement was gained yearly, so that ERP accuracy was improved. In May 1997 the LOD determination could be improved to about $0.04 \mathrm{~ms}$ by introducing orbital arcs with a length of 3 days, instead of 1 day. At the same time GFZ started to submit results for UT1, which are aligned on the first day of each week to IERS Bulletin A. The accuracy within the week is better than $0.1 \mathrm{~ms}$. The daily polar motion rates are estimated with an accuracy of about $0.2 \mathrm{mas} / \mathrm{d}$. This level was improved in March 1998 by switching from ITRF94 (with 13 fixed core sites) to ITRF96 (with 74 fixed core sites). This enlargement in the set of core sites stabilized the reference frame realization during the daily analysis. The largest improvement is in the rapid products, which have now an accuracy of 0.15 mas (Gendt et al. $1998 \mathrm{a}, \mathrm{b})$.

Geophysical processes accompanied by mass redistributions cause variations of polar motion and LOD. The impact of atmospheric, ocean and core dynamics on these variations are subjects of investigations. Variations of polar motion and the LOD can be estimated if sufficient information on the corresponding process is available. Höpfner (1995d, 1996a,b) investigated whether the AAM functions published by the IERS can completely explain the annual and semiannual periods of polar motion. It was found that the non-atmospheric excitation is much larger than expected. Imbalances in seasonal variations of the Earthatmosphere axial angular momentum budget were re-examined in (Höpfner 1995b, 1996b, $1997 \mathrm{a}, \mathrm{b}$, and $1998 \mathrm{a}, \mathrm{b})$ on the basis of LOD and AAM series. The results derived from different LOD and AAM systems show the extent to which the variations reflect systematic differences and significant signals, respectively. The imbalances in seasonal variations may be largly eliminated, if the missing impact of wind from the $10^{-1} \mathrm{hPa}$ is included in the solid Earth-atmosphere axial momentum budget. At the current level of uncertainty, the Earth's axial angular momentum budget for the annual oscillation is most likely closed with the missing wind contribution alone and for the semiannual oscillation with the missing wind contribution and the hydrological contribution. An excitation source such as the Antarctic Circum-Polar current is not negligible at lower levels of certainty. The influence of ENSO on variations of LOD and corresponding AAM terms was studied. Their temporal variations are partly related to variations of the SOI (Höpfner 1999).

Core dynamics is said to be a candidate for decade variations of the Earth's rotation, but an estimation of the atmospheric excitation function of polar motion and the LOD for the interval of time 1900-1989 shows that long-term influences of the atmosphere can be expected. In (Jochmann, Greiner-Mai 1996 and Geiner-Mai, Jochmann 1998) the studies of long term variations of LOD focused on explaining the climate cycles contained in this time series. It was found that the 11 and 22-year cycles are mainly caused by variations of atmospheric circulation, while the remaining decade fluctuations are probably produced by core-mantle coupling. Investigating the variations of polar motion for the period 1900-1989 a number of climate cycles could be identified (Jochmann 1998b). These cycles were also found in the amplitude modulations of the Chandler-wobble. Comparing the amplitude spectra of polar motion with those of the corresponding excitation function, it was found that the 11-year period and the 80-year Gleisberg cycle are partly caused by atmospheric mass redistributions. Considering inner core motion as a possibility for exciting decade fluctuations of polar motion it could be proved that the $11,22,30$, and 80 years cycles are mainly caused by this process. The phase shift between inner core excitation and polar motion supports this result because it corresponds with the Alfven wave velocity in the outer core. This indicates that climate cycles in Earth rotation seem to be more related to core dynamics than to atmospheric circulation.

Investigations of magnetic core-mantle coupling were continued using improved series of variations of the LOD and the geomagnetic field. Variations of LOD were reduced because of atmospheric effects (Greiner-Mai 1995, Jochmann and Greiner-Mai 1995,1996; GreinerMai and Jochmann 1998). The coupling model works with a conductivity in the lower mantle which is reduced by about $14 \%$. A new LOD series was constructed using modern 
and historical data (Liao and Greiner-Mai 1998) and compared with coupling parameters derived from the spherical harmonic coefficients of the International Geomagnetic Reference Field. Relations between geomagnetic field variations and polar motion were studied, taking into account the hypothesis of inner core motion (Greiner-Mai 1997). It was found that the coincidence between both axes is given if the magnitude of the angular velocity of the inner core is sufficiently high and the current system is concentrated in a thin sheet at the outer-inner core boundary. In (Greiner-Mai et al. 1998) the influence of inner core motion on polar motion and on the gravity field was calculated, using the hypothesis and standard density models of the Earth. Calculations of the gravity potential show that the rotation of the inner core causes gravity changes detectable by modern satellite missions (Reigber et al. 1997 and Tapley 1997).

The increasing accuracy of satellite missions allows an improved determination of the gravity field and its temporal variation. Jochmann (1997b) discussed whether the combined use of temporal variations of the gravity and the Earth's rotation reduces the ambiguity of the inverse solution for identifying global geophysical processes. An example of an inverse solution is given in (Jochmann 1999a,b). Since the annual wobble of polar motion can not be completely explained by atmospheric mass redistributions, an inverse solution was used to identify the unknown excitation. As a possible candidate for this excitation the seasonally varying water storage was chosen.

The influence of ocean tides on polar motion and the LOD were treated (Seiler and Wünsch 1995 and Wünsch 1995, 1997). Based on the Seiler model of ocean tides, more complete results for tidal induced periodic changes of Universal Time and polar motion were given. 24 smaller tidal constituents were added to the 10 previously published.

\subsection{NASA Goddard Space Flight Center}

The CORE program (Continuous Observations of the Rotation of the Earth) is designed to provide continuous, high accuracy measurements of Earth orientation using VLBI. CORE is a cooperative international scientific observing program, led by the VLBI group at NASA Goddard Space Flight Center. The goal of the program is to provide daily and subdaily values for polar motion and UT1 with accuracy goals of sub-microsecond UT1 and 25 micro-arcsecond pole position. With the start of the CORE program the geodetic VLBI community shifted its focus from measuring contemporary plate tectonics, to measuring Earth rotation. The CORE program has been evolving since 1997 and the full program is anticipated to be in place by 2003 . The observing program uses different VLBI networks on different days of the week. Observing began in January, 1997, with sessions designed to determine whether the different VLBI networks that would observe on different weekdays would give the same EOP result. The second goal of the initial sessions was to demonstrate the ability and performance of stations that would participate in the full CORE program.

The CORE-A sessions were run simultaneously with NEOS (a program of the U. S. National Earth Orientation Service) sessions to try to answer the first question. The differences between two measurement series are still not fully understood and are under active investigation. For the CORE-B sessions compared to NEOS, the $x$ - and $y$-pole differences are not significantly different from zero, but the UT1 bias is 10 sigma. The next step for CORE is to expand the number of observing days per week. Until the Mark IV correlator is available, the number of observing days that can be processed is limited.

During the past three years the CORE program has been achieving 3.5 microsec for UT1 and 100 micro-as for pole position. As the Mark IV correlator begins operation, it will be possible to use weaker sources because of the increased sensitivity of the Mark IV system. The next step toward increased accuracy will require improvements in the observing strategy to take advantage of the increased sensitivity. The CORE program will evolve toward providing daily and sub-daily VLBI measurements of UT1 and pole position. More continuous VLBI measurements will enable detailed comparisons of GPS and VLBI polar motion results, leading to better understanding of systematic effects in both techniques. 


\subsection{Astronomical Institute, Academy of Sciences of the Czech Republic, Faculty of Civil Engineering, Czech Technical University, Prague}

The Department of Dynamical Astronomy, Astronomical Institute of the Academy of Sciences of the Czech Republic collected the values of latitude and Universal Time observed by classical astrometric methods since the beginning of the century at observatories all over the world in order to prepare a new solution of EOP. These values, based on individual star observations, were converted into the International Celestial Reference System (ICRS) adopted by the IAU in 1997. The Hipparcos Catalogue was used as a principal realization of this system in optical wavelengths, and the task was done in close cooperation with the Department of Geodesy, Faculty of Civil Engineering in Prague.

Before the definitive Hipparcos Catalogue became available in 1997, either local star catalogues or preliminary versions of the Hipparcos Catalogue were used, mostly to test the algorithms and software package. The preliminary solutions were used to analyse the long-periodic behaviour of the Earth's orientation in space (Vondrak 1997a, Vondrak, Ron and Pesek 1997a, 1997b, Bizouard, Capitaine, Ron and Vondrak 1997). They developed an original method in which the rotating Earth served as an intermediary between the optical and extragalactic reference frames (Vondrak 1996, Vondrak, Ron, Pesek and Cepek 1996, Vondrak and Ron 1997c, Vondrak, Ron and Pesek 1997c). The results were then combined with those of other groups using different methods (Kovalevsky et al. 1997) to orient the Hipparcos Catalogue.

Statistically significant drifts in the residuals of individual stars, interpreted as errors of proper motions in the Hipparcos Catalogue, were found not only for multiple systems but also for some stars denoted as single. Corrections were applied prior to the solution (Pesek and Ron 1998). The solution, based on the observations made with 48 instruments at 31 observatories in the interval 1899.7-1992.0 (Vondrak, Ron, Pesek and Cepek 1997a, 1997b, Vondrak, Pesek, Ron and Cepek 1998) was then subject to further analysis in order to find the characteristics of the long-periodic behavior of the Earth's orientation (Vondrak and Ron 1997b, Korsun, Kurbasova and Vondrak 1998, Vondrak 1998a, 1998b, 1998c, 1998e, 1999b, Vondrak, Ron, Pesek and Cepek 1998, Gross and Vondrak 1999). The analysis reconfirmed the secular polar motion of about 3mas/yr in the direction of roughly $75^{\circ}$ West. Corrections of nine most dominant nutation terms (mostly consistent with those found from VLBI observations) were found. The correction of the precession constant found from these observations (covering nearly a century) is however two times smaller than that found from VLBI observations. A possible explanation is either non-linear behavior of precession or a slow residual rotation of the Hipparcos system with respect to ICRS of about $0.65 \mathrm{mas} / \mathrm{yr}$ around the y-axis. The determination of UT1 disclosed a strange systematic change of trend (when compared with the IERS combined solution), equivalent to a small step of about -6 microseconds in LOD that occurred around 1980-1983. Another solution is being prepared using more observations obtained from the observatories already used in the previous solution (Blagoveschtschensk, Kharkov) and including the data from two more observatories (Mount Stromlo and Jozefoslaw). The optical astrometric solution of EOP was combined with the space geodesy solution SPACE97 prepared at JPL. Secular and long-periodic polar motion of this combined solution was then studied (Vondrak 1999a, $1999 \mathrm{~b}$ ). It was found that the large change of phase of the Chandler wobble that occurred in 1920-1940 could be explained by a very small excitation of roughly Chandlerian frequency, preceding the polar motion component by about $90^{\circ}$ and lasting only between 1916 and 1950.

Theoretical nutation was calculated indirectly from the new and highly accurate tidal potential development derived by Hartmann and Wenzel in 1995 (Hartmann, Soffel and Ron 1999). Except for the long-periodic and largest nutation terms this method can compete with the traditional method and is a useful validation of the existing nutation series.

Studies were begun concerning the combination of the results of EOP provided by space geodetic methods. It was found that different techniques have different accuracy at different frequencies (Vondrak 1998d). Therefore it was proposed to divide the results 
obtained from different analysis centers into several frequency windows, apply different weights to combine them separately at each window, and then to add them together to obtain the final combination.

\subsection{Instituto de Astronoma y Geodesia, Madrid}

Work was accomplished in the areas of the theoretical study of quasi-diurnal and subdiurnal nutations coming from the harmonics of degree 3 and 4 of the geopotential and the corresponding numerical coefficients, Tables REN-2000 of the nutation for a rigid Earth model, starting from Hamiltonian theory, with a level of truncation at $0.1 \mu$ as, and the differents sets of variables involved in the Earth's rotation problem.

\subsection{Jet Propulsion Laboratory (JPL)}

Theoretical work is being done on the orientation and rotation of the Earth (James Williams). For obliquity rate, the total tidal contribution is small (Different tidal components tend to cancel.), the other contributions have been reevaluated, and the total rate in space is -0.270 mas/yr. Polar motion investigations focused on the effect of long-period ocean tides on polar motion (Gross et al., 1998) as well as on the secular drift in the pole path as evidenced by the new Hipparcos optical astrometric series (Gross and Vondrak 1999).

LOD changes are predominantly caused by changes in the angular momentum of atmospheric winds with atmospheric pressure changes contributing about $5 \%$ to the observed LOD changes. Including OAM significantly improves the coherence between the LOD and the AAM and helps close the angular momentum balance between the atmosphere, oceans, and solid Earth (Marcus et al., 1999). The effect of the recent 1997-98 ENSO event is clearly evident in the LOD observations, which are highly correlated with the SOI and interannual AAM variations. Robust V-like globally coherent structures originating in the tropics and propagating poleward are evident in latitudinally banded AAM data from the NCEP reanalyses (Dickey et al., 1999).

Earth rotation parameters are measured and predicted at JPL in support of tracking and navigating spacecraft in deep space. Improvements in the knowledge of the Earth's rotation directly lead to improvements in spacecraft tracking and navigation, enabling modern navigation techniques. Twice-per-week, rapid Earth orientation measurements taken by the techniques of VLBI, SLR, and GPS are collected and combined using the Kalman Earth Orientation Filter developed for this purpose. As part of this operational combination and prediction of Earth orientation parameters, annual combinations of the most accurate Earth orientation measurements have continued to be generated, the methodology employed being described by Gross et al. (1998) and Gross (1999a, 1999b).

Dennis D. McCarthy President of the Commission 